
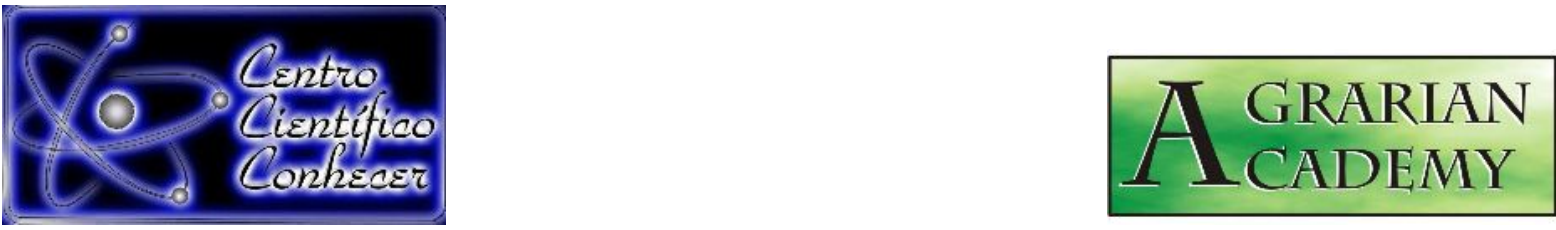

\title{
DESEMPENHO DE PRODUTOS ORBITAIS NA ESTIMATIVA DO BALANÇO HÍDRICO DA BACIA DO RIO XINGU
}

\author{
Charles Caioni
}

Doutor em Ecologia e Conservação pela Universidade Estado de Mato Grosso, Alta

Floresta/MT, Brasil, (charlescaione@hotmail.com)

Recebido em: 15/06/2021 - Aprovado em: 15/07/2021 - Publicado em: 30/07/2021

DOI: 10.18677/Agrarian_Academy_2021A2

\begin{abstract}
RESUMO
Conhecer o balanço hídrico de uma região é importante, tanto em aspectos ecológicos quantos econômicos. Entretanto, devido à dimensão territorial e dificuldade de acesso de algumas áreas é necessário realizar estimativas a partir de imagens orbitais. Nessa conjectura, objetivamos identificar quais combinações de produtos orbitais são mais adequadas para estimar o balanço hídrico em pequena e média escala no Sul da Amazônia. Os produtos utilizados nas diferentes combinações foram: 3B42_TRMM (sensor Tropical Rainfall Measuring MissionTRMM), MOD16A2, (sensor Moderate Resolution Imaging SpectroradiometerMODIS) e GRACE (Gravity Recovery and Climate Experiment). Enquanto as métricas de avalição foram: coeficiente de determinação $\left(R^{2}\right)$, erro médio quadrático (RMSE), viés (BIAS) e Percent Bias (PBIAS). Evidenciamos que a combinação de produtos CHIRPS, GRACE e MOD16 coleção 005 é a mais adequada para estimar o balanço hídrico, em ambas as escalas. Entretanto, a combinação TRMM, GRACE e MOD16 (col. 006) também demonstrou resultados satisfatórios para estimativas em pequena escala. Todas as estimativas exibiram um nítido comportamento sazonal, entretanto esse padrão não foi observado para os dados registrados em campo. Constatamos ainda que a evapotranspiração é um importante componente do balanço hidrológico na bacia, demonstrando assim a importância da conservação da vegetação local.
\end{abstract}

PALAVRAS-CHAVE: Amazônia Meridional, Bacia hidrográfica, controle de qualidade. 


\title{
PERFORMANCE OF ORBITAL PRODUCTS IN THE ESTIMATION OF THE XINGU RIVER BASIN WATER BALANCE
}

\begin{abstract}
Knowing the water balance of a region is important, both in ecological and economic aspects. However, due to the territorial dimension and difficulty in accessing some areas, it is necessary to make estimates based on orbital images. In this conjecture, we aim to identify which combinations of orbital products are most suitable for estimating water balance on a small and medium scale in Southern Amazonia. The products used in the different combinations were: 3B42_TRMM (Tropical Rainfall Measuring Mission-TRMM sensor), MOD16A2, (Moderate Resolution Imaging Spectroradiometer-MODIS sensor) and GRACE (Gravity Recovery and Climate Experiment). While the assessment metrics were: determination coefficient $\left(R^{2}\right)$, mean square error (RMSE), bias (BIAS) and Percent Bias (PBIAS). We show that the combination of CHIRPS, GRACE and MOD16 collection 005 products is the most suitable for estimating water balance, on both scales. However, the combination TRMM, GRACE and MOD16 (col. 006) has also shown satisfactory results for smallscale estimates. All estimates showed a clear seasonal behavior, however this pattern was not observed for data recorded in the field. We also found that evapotranspiration is an important component of the hydrological balance in the basin, thus demonstrating the importance of conserving local vegetation.
\end{abstract}

KEYWORDS: Quality control, Southern Amazonia, watershed.

\section{INTRODUÇÃO}

A disponibilidade de agua é um fator primordial para a existência de vida em qualquer local do planeta (ANTUNES;CUPOLILLO, 2018). Assim, conhecer o comportamento temporal do balanço hídrico de uma região é extremamente importante, tanto em aspectos ecológicos quanto econômicos (SANTOS et al., 2018, Cavalcante et al., 2019). Do ponto de vista ecológico, a quantidade de água que permanece em um sistema (bacia hidrográfica) pode determinar a estrutura e composição da vegetação, uma vez que a disponibilidade hídrica atua como um filtro de espécies. Do ponto de vista econômico, a compreensão do balanço hídrico pode influenciar na data de plantio (ROCHA et al., 2020), bem como na capacidade de geração de energia de uma usina hidrelétrica.

No Sul da Amazônia estimar o balanço hídrico a partir de dados coletados localmente é algo oneroso e quase inviável, isso porque seria necessário um grande número de estações meteorológicas para cobrir o território amazônico (MOTTA PACA et al., 2020). Nessa conjectura o uso de dados de precipitação, evapotranspiração e água armazenada no solo provenientes de sensores remotos pode ser uma alternativa (ERAZO et al., 2018; LEVY et al 2018; WENG et al., 2018; MOLINA et al., 2019). Entre os produtos mais utilizados para a precipitação estão os do sensor Tropical Rainfall Measuring Mission (3B42_TRMM) (HUFFMAN et al., 2007; Yi et al., 2018; FANG et al., 2019; ABDULRAZZZAQ, 2020;) e do Climate Hazards Group Infra Red Precipitation with Station Data (CHIRPS) (DINKU et al., 2018; RIVERA et al., 2018; CAVALCANTE et al., 2020; LARBI et al., 2020; SHEN et al., 2020). Para a evapotranspiração tem-se o sensor Moderate Resolution Imaging Spectroradiometer (MODIS, produto MOD16A2 coleção 005) (MU et al., 2011), 
enquanto para a água armazenada no solo há o Gravity Recovery and Climate Experiment (GRACE) (WARD, 2004).

Embora estimar o balanço hídrico a partir de imagens orbitais seja uma alternativa, existem incertezas quantos a acurácia dessas estimativas (NOGUEIRA et al., 2018), sobretudo para áreas pequenas na Amazônia. Isso porque, além da maior parte dos produtos disponíveis apresentarem resolução grosseira, a grande maioria foram calibrados com dados provenientes em sua maior parte da América do Norte.

Tendo em vista a importância da compreensão do balanço hídrico, bem como as incertezas na acurácia das estimativas atuais, focou-se em identificar quais combinações de produtos orbitais são mais adequadas para estimar o balanço hídrico em pequena e média escala no Sul da Amazônia.

\section{Área de estudo}

\section{MATERIAL E MÉTODOS}

Esta pesquisa compreendeu duas escalas. Em pequena escala considerou-se a sub-bacia do Rio Comandante Fontoura que faz parte da bacia do Rio Xingu. Distribuída entre os estados do Pará e de Mato Grosso, os $13.569 \mathrm{~km}^{2}$ da sub-bacia abrigam grandes áreas de florestas nativa, ao mesmo tempo que é responsável por elevada produção de carne e grãos (COE et al. 2017). Em média escala foi considerada a bacia do Rio Xingu (figura 1), que compreende a região Sul da Amazônia. Sua área de drenagem é de $\sim 170.000 \mathrm{~km}^{2}$ (GARCIA et al., 2019), com cerca de $2.000 \mathrm{~km}$ de comprimento.

\section{Produtos utilizados}

- Precipitação (CHIRPS e TRMM)

A série histórica de precipitação (2002 a 2018) foi obtida a partir dos produtos CHIRPS (DINKU et al., 2018; RIVERA et al., 2018), e TRMM (HUFFMAN et al., 2007). Para o produto TRMM, foram utilizados dados provenientes do algoritmo 3B42, versão 7. Os dados utilizados do produto CHIRPS consistem na versão 2.0, com resolução temporal de 1 dia e espacial de $0.05^{\circ}$.

\section{- Produto MOD16A2}

Os dados de evapotranspiração foram obtidos de duas series temporais do produto MOD16A2. A coleção 005 (resoluções espaciais de $0.5 \mathrm{~km}$ ) de 2002 a 2014, e a coleção 006 (resoluções espaciais de $0.5 \mathrm{~km}$ ) de 2002 a 2018.

- Dados Shuttle Radar Topographic Mission (SRTM)

O modelo digital de elevação (MDE) foi gerado a partir de imagens proveniente da missão Shuttle Radar Topographic Mission (SRTM), refinados para 30 metros pelo projeto TOPODATA (Banco de Dados Geomorfométricos do Brasil) (VALERIANO; ROSSETTI, 2011) e disponibilizadas pelo Instituto Nacional de Pesquisas Espaciais (INPE).

\section{- Produto GRACE}

Os dados de presença de agua no solo foram obtidos a partir da série histórica (2003 a 2014) do satélite GRACE (Gravity Recovery And Climate Experiment) (WARD, 2004). 


\section{- Balanço hídrico observado}

Considera-se como balanço hídrico observado os dados reais de vazão hídrica coletados nas estações fluviométricas do Rio Comandante Fontoura e de AltamiraPA, ambas pertencentes a Agência Nacional de Águas - ANA.

\section{Procedimentos metodológicos}

Para alcançar o objetivo realizaram-se quatro análises: $(I)$ inicialmente, delimitouse a área de drenagem (sub bacia) do Rio Comandante Fontoura; (ii) em seguida, a partir de imagens orbitais, estimou-se o balanço hidrológico para a sub bacia do Rio Comandante Fontoura e para a bacia do Rio Xingu; (iii) por último, foi identificada qual combinação de produtos orbitais é a mais adequada para estimar o balanço hidrológico em ambas unidades hidrográficas

\section{I) Delimitação da área de drenagem}

A área de drenagem da sub bacia do Rio Comandante Fontoura foi obtida a partir do modelo digital de elevação (MDE), construído de imagens SRTM (FARR et al., 2007). No software ArcMap 10.8 (ESRI, 2021), o MDE foi obtido a partir dos respectivos procedimentos: eliminação das depressões espúrias, geração das direções de fluxo, geração do fluxo acumulado, e delimitação automática da bacia (FRAN et al, 2018; MACÊDO; SURYA, 2018; SILVA et al., 2018).

\section{II) Estimativa do balanço hídrico.}

A estimativa do balanço hídrico da bacia do Rio Xingu, foi gerada a partir de diferentes combinações dos produtos MOD16 (coleções 5 e 6), TRMM_3B42 (versão 7), CHIRPS (versão 2.0) e GRACE. Primeiramente, calcularam-se as somas anuais das séries históricas de cada produto orbital. Neste sentido, consideraram-se respectivamente os meses de outubro e setembro como início e fim de cada hidroperíodo, a fim de reduzir o efeito tardio que ocorre entre a precipitação e a recarga dos rios. O balando hidrológico foi obtido conforme equação abaixo.

$$
Q=P P T-E T-W
$$

Onde, $Q$ representa o balanço hidrológico estimado; $E T$ consiste na evapotranspiração e $W$ a água contida no solo.A partir do pacote raster $\mathrm{R}$ (HIJMANS et al., 2014) realizou-se ainda, a extração e soma dos valores de $Q$ contidos dentro de cada sub bacia, para posteriormente compará-los com os valores observados.

\section{III) Validação do balanço hídrico estimado}

A fim de identificar qual combinação de produtos orbitais é a mais adequada para estimar o balanço hídrico na bacia do Rio Xingu, foram comparados os dados estimados com os observados nas estações fluviométricas.

O coeficiente de determinação $\left(R^{2}\right)$, erro médio quadrático (RMSE) e viés (BIAS) e Percent Bias (PBIAS), foram obtidos a partir das seguintes equações (COSTA et al., 2019).

$$
\mathrm{R}^{2}=\frac{(S x y)^{2}}{S x x S y y}
$$


Sendo, Sxx e Syy - Soma dos quadrados dos desvios das médias; Sxy - Soma dos produtos cruzados dos desvios de $x$ e $y$.

$$
\begin{aligned}
& R M S E \sqrt{(V f t-V \text { orb })^{2} / N} \\
& \text { BIAS }=(V f t-V \text { orb }) / N \\
& \text { PBIAS }=\frac{\text { Bias }}{\left(\frac{1}{n}\right) \Sigma V f t} * 100
\end{aligned}
$$

Onde: $V$ ft representa os dados observados da Torre de Fluxo, $V$ orb os dados do produto orbitais (MOD16 e TRMM_3B42) e $N$ é o número de medidas. Quanto mais próximo de zero os valores de RMSE e BIAS, maior a acurácia dos dados modelados com os observados. Valores negativos e positivos de BIAS representam respectivamente, subestimação e superestimação das variáveis estimadas.

\section{RESULTADOS E DISCUSSÃO}

A acurácia da estimativa do balanço hídrico variou de acordo com a escala (pequena e média) e os produtos utilizados (Tabela 1). Em pequena escala (Sub bacia do Rio Comandante Fontoura) o balanço hídrico estimado com os produtos CHIRPS, GRACE e MOD16 (col. 005) demonstrou maior acurácia $\left(R^{2}=0.74\right.$, BIAS=121.83, RMSE = 45.57), seguido pela combinação TRMM, GRACE e MOD16A2 (coleção 006). Cabe ressaltar que esta última combinação demonstrou necessitar de calibração, mediante superestimativa do balanço em 36\%. Em média escala também constatou-se que o uso conjunto do CHIRPS, GRACE e MOD16 (Col. 005) é mais indicado para estimar o balanço hídrico na Bacia do Rio Xingu $(\mathrm{BIAS}=209.90, \mathrm{RMSE}=42.84)$. Com $\mathrm{R}^{2}$ de 0.84 os dados balanço estimado exibiu forte relação com o observado. O balanço estimado com os produtos TRMM, GRACE e MOD16 (col. 005) também se assemelhou ao observado pela ANA $\left(R^{2}=\right.$ 0.50 e RMSE $=55.92)$, embora o modelo também tenha superestimado o balanço em mais de 50\% ( 274 mm/ano) (Figura 1). 
TABELA 1. Comparação entre o balanço hídrico observado e o estimado na Bacia do Rio Xingu.

\begin{tabular}{|c|c|c|c|c|c|c|c|c|c|c|c|}
\hline $\begin{array}{c}\text { Unidade } \\
\text { hidrográfica }\end{array}$ & Estação & Latitude & Longitude & $\begin{array}{c}\text { Área da } \\
\text { drenagem }\end{array}$ & $R^{2}$ & $\begin{array}{l}\text { Valor } \\
\text { de } p\end{array}$ & $N$ & $\begin{array}{c}\text { BIAS } \\
\text { (mm/ano) }\end{array}$ & PBIAS & RMSE & Produtos \\
\hline $\begin{array}{l}\text { Bacia do rio } \\
\text { Xingu }\end{array}$ & Altamira & -3.2147 & -52.2122 & 448000 & $\begin{array}{c}0.8 \\
4\end{array}$ & 0.00 & 13 & 209.90 & 38.24 & 42.84 & $\begin{array}{l}\text { GRACE, CHIRPS } \\
\text { e MOD16 (col. } \\
\text { 005) }\end{array}$ \\
\hline $\begin{array}{l}\text { Bacia do rio } \\
\text { Xingu }\end{array}$ & Altamira & -3.2147 & -52.2122 & 448000 & $\begin{array}{c}0.2 \\
9\end{array}$ & 0.25 & 13 & 226.43 & 41.25 & 47.20 & $\begin{array}{l}\text { GRACE, CHIRPS } \\
\text { e MOD16 (col. } \\
\text { 006) }\end{array}$ \\
\hline $\begin{array}{l}\text { Bacia do rio } \\
\text { Xingu }\end{array}$ & Altamira & -3.2147 & -52.2122 & 448000 & $\begin{array}{c}0.5 \\
0\end{array}$ & 0.03 & 13 & 273.95 & 49.91 & 55.92 & $\begin{array}{l}\text { GRACE, TRMM e } \\
\text { MOD16 (col. 005) }\end{array}$ \\
\hline $\begin{array}{l}\text { Bacia do rio } \\
\text { Xingu }\end{array}$ & Altamira & -3.2147 & -52.2122 & 448000 & $\begin{array}{c}0.1 \\
8\end{array}$ & 0.50 & 13 & 354.71 & 64.62 & 72.40 & $\begin{array}{l}\text { GRACE, TRMM } \\
\text { e MOD16 (col. } \\
\text { 006) }\end{array}$ \\
\hline $\begin{array}{l}\text { Sub bacia do } \\
\text { Rio } \\
\text { Comandante } \\
\text { Fontoura }\end{array}$ & $\begin{array}{l}\text { Rio } \\
\text { Comanda } \\
\text { nte } \\
\text { Fontoura }\end{array}$ & -10.5547 & -52.1833 & 5430 & $\begin{array}{c}0.7 \\
3\end{array}$ & 0.01 & 6 & 121.83 & 23.21 & 45.57 & $\begin{array}{l}\text { GRACE, CHIRPS } \\
\text { e MOD16 (col. } \\
\text { 005) }\end{array}$ \\
\hline $\begin{array}{l}\text { Sub bacia do } \\
\text { Rio } \\
\text { Comandante } \\
\text { Fontoura }\end{array}$ & $\begin{array}{l}\text { Rio } \\
\text { Comanda } \\
\text { nte } \\
\text { Fontoura } \\
\end{array}$ & -10.5547 & -52.1833 & 5430 & $\begin{array}{c}0.2 \\
4\end{array}$ & 0.41 & 6 & 10.52 & 54.22 & 21.52 & $\begin{array}{l}\text { GRACE, CHIRPS } \\
\text { e MOD16 (col. } \\
\text { 006) }\end{array}$ \\
\hline $\begin{array}{l}\text { Sub bacia do } \\
\text { Rio } \\
\text { Comandante } \\
\text { Fontoura }\end{array}$ & $\begin{array}{l}\text { Rio } \\
\text { Comanda } \\
\text { nte } \\
\text { Fontoura }\end{array}$ & -10.5547 & -52.1833 & 5430 & $\begin{array}{c}0.6 \\
9\end{array}$ & 0.01 & 6 & 258.30 & 51.19 & 74.57 & $\begin{array}{l}\text { GRACE, TRMM e } \\
\text { MOD16 (col. } \\
\text { 005) }\end{array}$ \\
\hline $\begin{array}{l}\text { Sub bacia do } \\
\text { Rio } \\
\text { Comandante } \\
\text { Fontoura }\end{array}$ & $\begin{array}{l}\text { Rio } \\
\text { Comanda } \\
\text { nte } \\
\text { Fontoura } \\
\end{array}$ & -10.5547 & -52.1833 & 5430 & $\begin{array}{c}0.8 \\
4\end{array}$ & 0.00 & 6 & 188.08 & 36.48 & 50.27 & $\begin{array}{l}\text { GRACE, TRMM } \\
\text { e MOD16 (col. } \\
\text { 006) }\end{array}$ \\
\hline
\end{tabular}


FIGURA 1. Comparações entre o balanço hídrico observado e o estimado a partir dos produtos GRACE, CHIRPS, TRMM_3B42 e MOD16A2 (coleções 005 e 006). Painéis a direita representam a soma mensal, e a esquerda, a soma anual da precipitação. Os pontos representam medições e as linhas pontilhadas indicam correspondências 1: 1.

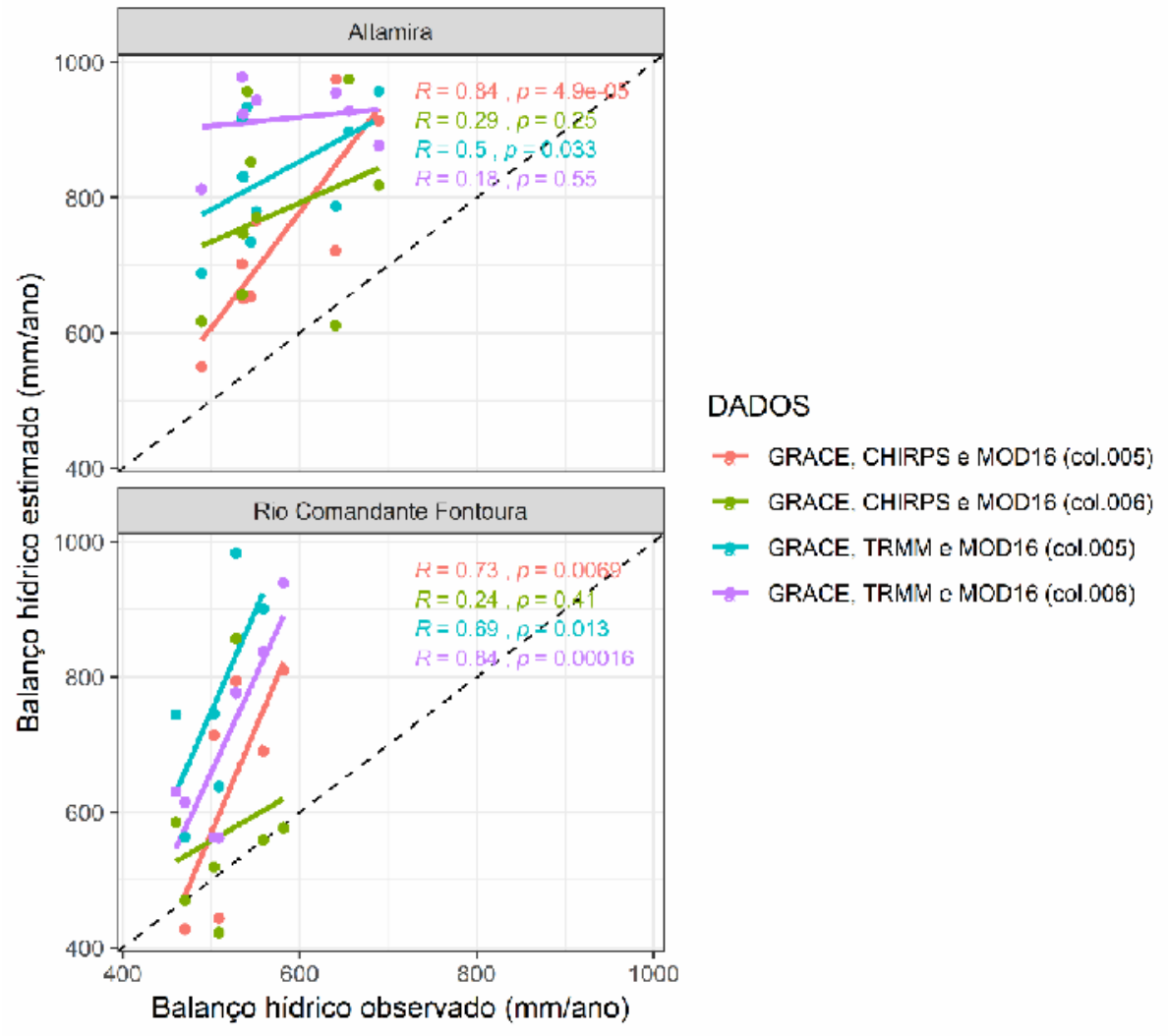

Fonte: próprio autor.

As estimativas realizadas a nível de bacia foram mais consistentes comparada a nível de sub bacia em decorrência de dois fatores, o primeiro é a grosseira resolução espacial dos produtos orbitais (CAIONI et al., 2020), como por exemplo o GRACE que é $1 \mathrm{grau}$. O segundo é o maior número de dados processados na Bacia do Rio Xingu, cerca de sete anos, o que pode ter reduzido a influência dos valores extremos (outliers) nos resultados.

Embora as estimativas do balanço hídrico estimado com o produto CHIRPS tenha demonstrado maior acurácia, houve superestimavas tanto em pequena quanto em grande escala. Esses erros podem também estar relacionados ao número de estações utilizadas para gerar o produto CHIRPS terem sido reduzidos nos últimos anos (PAREDES-TREJO et al., 2017).

Em ambas as escalas (pequena e média) o balanço hídrico estimado demonstrou um nítido comportamento sazonal (Figura 2). A nível de sub bacia este padrão foi ainda mais nítido, entretanto, não foi possível evidenciar distinção entre as diferentes estimativas do balanço hídrico. Em ambas as estimativas constataramse picos de máxima e mínima nos meses de junho e fevereiro, respectivamente. $O$ balanço hídrico observado também demonstrou uma variação sazonal, contudo, menos acentuada. Ao nível de bacia, o balanço hídrico estimado a partir dos produtos GRACE, TRMM e MOD16ET (col 005) demonstrou maiores amplitudes 
mensais (picos), principalmente nos meses de julho e fevereiro, quando as máximas e mínimas foram de $-43 \mathrm{~mm} / \mathrm{mês}$ e $151 \mathrm{~mm} / \mathrm{mês}$, respectivamente.

FIGURA 2. Sazonalidade do balanço hídrico estimado e observado em diferentes escalas. As áreas sombreadas denotam o intervalo interquartil.

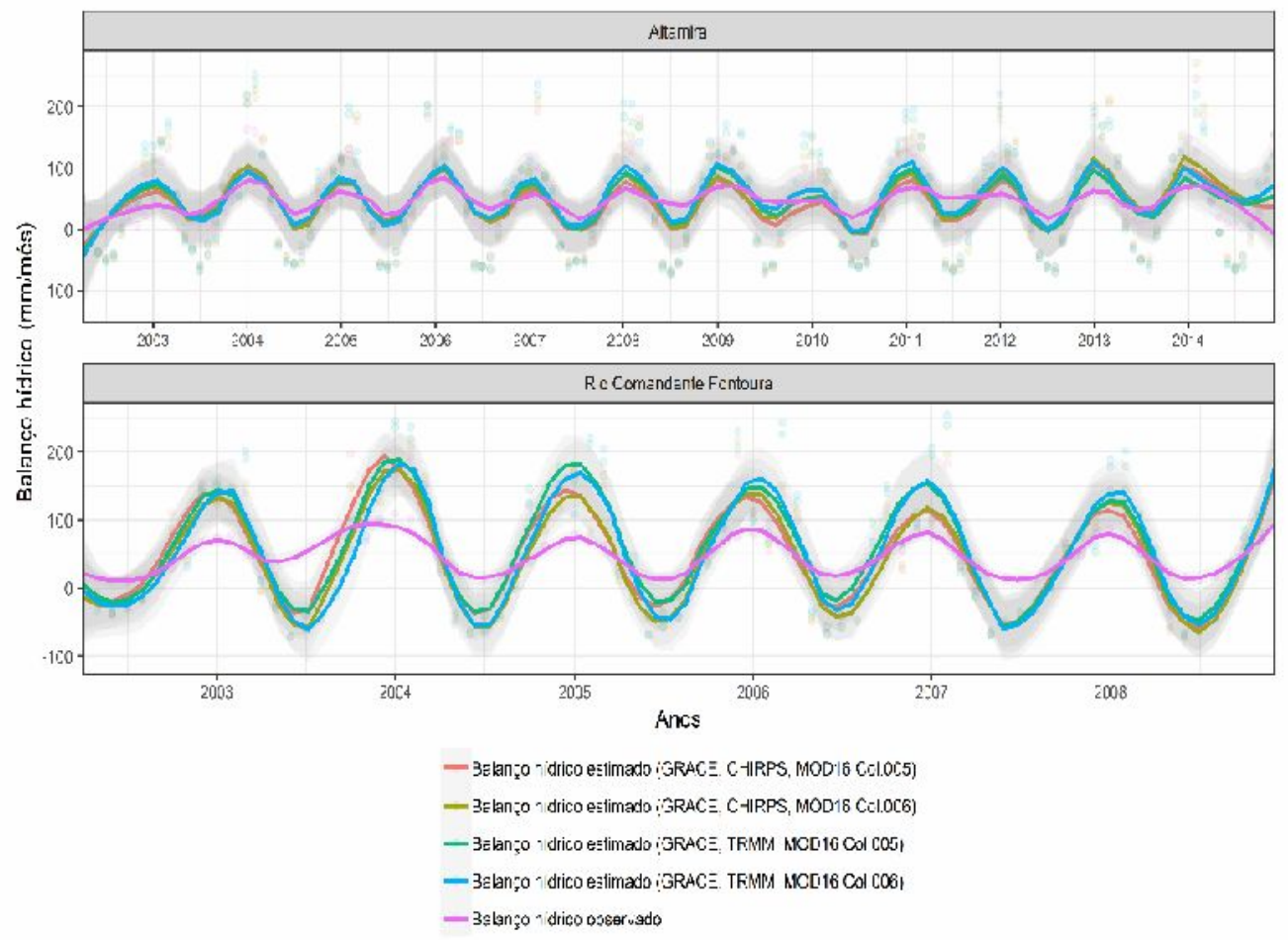

Fonte: próprio autor.

Tanto em pequena quanto em média escala, o balanço hídrico estimado demonstrou uma sazonalidade mais acentuada comparada ao observado, devido ao efeito tardio da seca sobre a vazão do Rio Xingu. Como a maior parte da água que entra na bacia do Rio Xingu é proveniente da chuva (SALATI, 1987), com o início da estação seca o saldo do balanço hídrico tende a reduzir, entretanto, essa redução não é imediata, e por ter início tardio acaba prolongando-se até os primeiros meses da estação chuvosa.

\section{CONCLUSÕES}

A combinação de produtos CHIRPS, GRACE e MOD16 coleção 005 é a mais adequada para estimar o balanço hídrico, tanto em pequena quanto em média escala. Contudo, estimativas realizadas a partir da combinação, TRMM, GRACE e MOD16 (col. 006) também demonstrou resultados satisfatórios, sobretudo em pequena escala.

Constatamos também, que considerável parcela de água que compõe o balanço hídrico na bacia na área estudada é proveniente do processo de evapotranspiração, demonstrando assim, a importância da preservação dos ambientes florestais, visto o seu papel na manutenção do ciclo hidrológico local e regional. 


\section{REFERÊNCIAS}

ABDULRAZZAQ, Z. T. The feasibility of using TRMM satellite data for missing terrestrial stations in Iraq for mapping the rainfall contour lines. Civ. Eng. Beyond Limits, v. 1, p. 15-19, 2020. Disponível em: < https://www.acapublishing.com/dosyalar/baski/CEBEL_2020_59.pdf.

doi.org/10.36937/cebel.2020.003.003 1>. Acessado em: 12 set. 2020.

ANTUNES, D. A.; CUPOLILLO, F. Análise do balanço hídrico climatológico decendial do parque estadual do rio doce-perd e entorno: climatologia de 2005-2015 comparada com o ano anômalo 2013-2014. Revista Brasileira de Climatologia, v. 1, $2018 . \quad$ Disponível em: < https://revistas.ufpr.br/revistaabclima/article/view/59778/36817>. Acessado em: 16 jan. 2021.

CAIONI, C.; SILVÉRIO, D. V.; MACEDO, M. N.; COE, M. T.; BRANDO, P. M. Droughts Amplify Differences Between the Energy Balance Components of Amazon Forests and Croplands. Remote Sensing, v. 12, n. 3, p. 525, 2020. Disponível em: < https://www.mdpi.com/2072-4292/12/3/525. 10.3390/rs12030525> Acesso em: 15 jun. 2020.

CAVALCANTE, R. B. L.; DA SILVA FERREIRA, D. B.; PONTES, P. R. M.; TEDESCHI, R. G.; DA COSTA, C. P. W.; DE SOUZA, E. B. Evaluation of extreme rainfall indices from CHIRPS precipitation estimates over the Brazilian Amazonia. Atmospheric Research, v. 238, p. 104879, 2020. Disponível em:.< https://www.sciencedirect.com/science/article/pii/S0169809519314073?casa_token= M2vXi6EAWloAAAAA:WZp7skZMBNukYuuolEOtgGM9HECzTTtE9vJAomuQBBrX9 PXSAek_7Vuv3RsSaVPu75YHt_NeemcL\#bb0085.

doi.org/10.1016/j.atmosres.2020.104879>. Acessado em: 04 jan. 2021.

CAVALCANTE, R. B. L.; PONTES, P. R. M.; SOUZA-FILHO, P. W. M.; DE SOUZA, E. B. Opposite effects of climate and land use changes on the annual water balance in the amazon arc of deforestation. Water Resources Research, v. 55, n. 4, p. 3092-3106, 2019. Disponível em:.< https://agupubs.onlinelibrary.wiley.com/doi/full/10.1029/2019WR025083. doi.org/10.1029/2019WR025083>. Acessado em: 07 set. 2020.

COSTA, J. C., PEREIRA, G., SIQUEIRA, M. E., DA SILVA CARDOZO, F., SILVA, V. V. Validação dos dados de precipitação estimados pelo CHIRPS para o Brasil. Revista Brasileira de Climatologia, v.24, p. 228-243, 2019. Disponível em::< https://revistas.ufpr.br/revistaabclima/article/view/60237.

doi.org/10.5380/abclima.v24i0.60237>. Acessado em: 01 maç. 2021.

DINKU, T.; FUNK, C.; PETERSON, P.; MAIDMENT, R.; TADESSE, T.; GADAIN, H.; CECCATO, P. Validation of the CHIRPS satellite rainfall estimates over eastern Africa. Quarterly Journal of the Royal Meteorological Society, v. 144, p. 292-312, 2018. Disponível em:< https://rmets.onlinelibrary.wiley.com/doi/full/10.1002/qj.3244. doi.org/10.1002/qj.3244>. Acessado em: 28 jan. 2020. 
ERAZO, B.; BOURREL, L.; FRAPPART, F.; CHIMBORAZO, O.; LABAT, D.; DOMINGUEZ-GRANDA, L.; MATAMOROS, D.; MEJIA, R. Validation of Satellite Estimates (Tropical Rainfall Measuring Mission, TRMM) for Rainfall Variability over the Pacific Slope and Coast of Ecuador. Water, v. 10, n. 2, p. 213, 2018. Disponível em: <https://www.mdpi.com/2073-4441/10/2/213. doi.org/10.3390/w10020213 >. Acessado em: 27 set. 2020.

ESRI, ArcGIS. 10.8. ArcGIS Desktop 10.8 quick start guide, 2021. Disponivel em: $<$ https://desktop.arcgis.com/en/arcmap/latest/get-started/setup/arcgis-desktop-quickstart-guide.htm >. Acessado em: 11 dez. 2020.

FANG, J.; YANG, W.; LUAN, Y.; DU, J.; LIN, A.; ZHAO, L. Evaluation of the TRMM $3 B 42$ and GPM IMERG products for extreme precipitation analysis over China. Atmospheric research, v. 223, p. 24-38, 2019. Disponível em: < https://www.sciencedirect.com/science/article/pii/S0169809518311499?casa_token=m_10TmddVgAAAAA:PM5azfxE3EmUhjjLtfF4iNuxkhyJ9rMyhs66J_rGT3-

J6u1ipCMXVngLnSMUPAy0ew5WGXqEL1tg.

doi.org/10.1016/j.atmosres.2019.03.001>. Acessado em: 25 dez. 2020.

FARR, T. G.; ROSEN, P. A.; CARO, E. The Shuttle Radar Topography Mission, Revista Geophys, v. 45, 2007. Disponível em: < https://agupubs.onlinelibrary.wiley.com/doi/epdf/10.1029/2005RG000183. 10.1029/2005RG00018 >. Acessado em: 11 jan. 2020.

FRAN, L. L. S. D.; ALMEIDA, A. D. O. D.; PENHA, A. D. L. T. D. Avaliação da qualidade dos modelos digitais de elevação ASTER e SRTM para o estado da Bahia. Revista Brasileira de Cartografia, v. 69, n. 9, 8 ago. 2018. Disponível em: <http://www.seer.ufu.br/index.php/revistabrasileiracartografia/article/view/44087>. Acessado em: 21 dez. 2020.

GARCIA, A. S.; DE VILELA, V. M.; RIZZO, R.; WEST, P.; GERBER, J. S.; ENGSTROM, P. M.; BALLESTER, M. V. R. Assessing land use/cover dynamics and exploring drivers in the Amazon's arc of deforestation through a hierarchical, multiscale and multi-temporal classification approach. Remote Sensing Applications: Society and Environment, v. 15, p. 100233, 2019. Disponível em:< https://www.sciencedirect.com/science/article/pii/S2352938519300291?casa_token= h-fwrBgW4hcAAAAA:t_3axY2Zc4sNT6muJA83zsKb_XPQxJMgSeWMb-8EIY7tQWmpwxxolPP6wrCyjJ0X8aTa2DiLY7i. doi.org/10.1016/j.rsase.2019.05.002>. Acessado em: 22 dez. 2020.

HIJMANS, R. J.; VAN ETTEN, J.; ETTEN, J.; MATTIUZZI, M. V.; SUMNER, M.; GREENBERG, J. A.; LAMIGUEIRO, O. P.; RACINE, E. B.; SHORTRIDGE, A. raster: Geographic data analysis and modeling. R package version, v. 2, p.15, 2014. Disponível em: <https://rdrr.io/cran/raster/>. Acessado em: 30 jan. 2020.

HUFFMAN, G. J.; BOLVIN, D. T.; NELKIN, E. J.; WOLFF, D. B.; ADLER, R. F.; GU, G.; HONG, Y.; BOWMAN, K. P. STOCKER, E. F. The TRMM multisatellite precipitation analysis (TMPA): Quasi-global, multiyear, combined-sensor precipitation 
estimates at fine scales. Journal of hydrometeorology, v. 8, n. 1, p. 38-55, 2007. Disponível em: <https://journals.ametsoc.org/view/journals/hydr/8/1/jhm560_1.xml. doi.org/10.1175/JHM560.1>. Acessado em: 11 set. 2020.

LARBI, I.; OBUOBIE, E.; VERHOEF, A.; JULICH, S.; FEGER, K. H.; BOSSA, A. Y.; MACDONALD, D.WATER balance components estimation under scenarios of land cover change in the Vea catchment, West Africa. Hydrological Sciences Journal, v. 65, n. 13, p. 2196-2209, 2020. Disponível em: < https://www.tandfonline.com/doi/abs/10.1080/02626667.2020.1802467. doi.org/10.1080/02626667.2020.1802467>. Acessado em: 13 dez. 2020.

LEVY, M. C.; LOPES, A. V.; COHN, A.; LARSEN, L. G.; THOMPSON, S. E. Land use change increases streamflow across the arc of deforestation in Brazil. Geophysical Research Letters, v.45, p. 3520-3530, 2018. Disponível em:< https://agupubs.onlinelibrary.wiley.com/doi/abs/10.1002/2017GL076526. doi.org/10.1 002/2017GL076526>. Acessado em: 11 dez. 2020.

MACÊDO, R. J. A.; SURYA, L. Comparação entre modelos digitais de elevação dos sensores SRTM e ALOS PALSAR para análise digital de terreno. Revista Contexto Geográfico, v. 3, n. 6, p. 47-55, 2018. Disponível em: < https://www.seer.ufal.br/index.php/contextogeografico/article/view/6968. doi.org/10.28998/contegeo.v3i6.6968>. Acessado em: 02 set. 2020.

MOLINA, R. D.; SALAZAR, J. F.; MARTÍNEZ, J. A.; VILLEGAS, J. C.; ARIAS, P. A. Forest-induced exponential growth of precipitation along climatological wind streamlines over the Amazon. Journal of Geophysical Research: Atmospheres, v. 124, n. 5, p. 2589-2599, 2019. Disponível em: < https://agupubs.onlinelibrary.wiley.com/doi/full/10.1029/2018JD029534. doi.org/10.1029/2018JD029534>. Acessado em: 16 jan. 2021.

MOTTA PACA, V. H.; ESPINOZA-DÁVALOS, G. E.; HESSELS, T. M.; MOREIRA, D. M.; COMAIR, G. F.; BASTIAANSSEN, W. G. The spatial variability of actual evapotranspiration across the Amazon River Basin based on remote sensing products validated with flux towers. Ecological Processes, v. 8, n. 1, p. 1-20, 2019. Disponível.em:.<https://ecologicalprocesses.springeropen.com/articles/10.1186/s137 17-019-0158-8. doi.org/10.1186/s13717-019-0158-8>. Acessado em: 02 maç. 2021.

MU, Q., ZHAO, M., RUNNING, S.W. Improvements to a MODIS global terrestrial evapotranspiration algorithm. Remote Sensing of Environment, v. 115, n. 8, p. 1781 -

1800,.2011..Disponível.em:.<https://www.sciencedirect.com/science/article/abs/pii/S0 034425711000691. doi.org/10.1016/j.rse.2011.02.019>. Acessado em: 16 maç. 2021.

NOGUEIRA, S. M. C.; MOREIRA, M. A.; VOLPATO, M. M. L. Evaluating Precipitation Estimates from Eta, TRMM and CHRIPS Data in the SouthSoutheast Region of Minas Gerais State-Brazil. Remote Sensing, v. 10, n. 2, p. 313, 2018. Disponível em:.<https://www.mdpi.com/2072- 
4292/10/2/313https://www.mdpi.com/2072-4292/10/2/313.

doi.org/10.3390/rs10020313>. Acessado em: 06 set. 2020.

PAREDES-TREJO, F. J.; BARBOSA, H. A.; LAKSHMI KUMAR, T. V. Validating CHIRPS-based satellite precipitation estimates in Northeast Brazil. J. AridEnviron, v. 139, p. 26-40, 2017. Disponível em: < https://www.sciencedirect.com/science/article/abs/pii/S014019631630235X. doi.org/10.1016/j.jaridenv.2016.12.009 >. Acessado em: 15 nov. 2020.

RIVERA, J. A.; MARIANETTI, G.; HINRICHS, S. Validation of CHIRPS precipitation dataset along the Central Andes of Argentina. Atmospheric Research, v. 213, p. 437-449, $2018 . \quad$ Disponível em:.< https://www.sciencedirect.com/science/article/pii/S0169809518301042?casa token= zW97SxvsRkwAAAAA:LqDEfEzSCWV4INJd9TOUx2QdQt0LO1wBal2LC4JOgh1JN O9Y2Q6_q-tKYHWNdf3EeT156G3vcqZq. doi.org/10.1016/j.atmosres.2018.06.023>. Acessado em: 11 maç. 2021.

ROCHA, N. S.; CARVALHO, C. M.; KÄFER, P. S.; IGLESIAS, M. L.; DA SILVA, S. C. S.; ROLIM, S. B. A.; CRUZ, R. C. Análise do balanço hídrico climatológico da subbacia hidrográfica do alto Camaquã-RS. Geo UERJ, n. 37, 2020. Disponível em: <https://www.e-publicacoes.uerj.br/index.php/geouerj/article/view/37723. 10.12957/geouerj.2020.37723>. Acessado em: 16 maç. 2021.

SALATI, E. The forest and the hydrological cycle. The Geophysiology of Amazonia. RE Dickinson, Ed. 1987.

SANTOS, V.; LAURENT, F.; ABE, C. MESSNER, F. Hydrologic response to land use change in a large basin in Eastern Amazon. Water, v. 10, n. 4, p. 429, 2018. Disponível.em:.<https://www.mdpi.com/2073-

4441/10/4/429..https://doi.org/10.3390/w10040429>. Acessado em: 16 maç. 2021.

SHEN, Z.; YONG, B.; GOURLEY, J. J.; QI, W.; LU, D.; LIU, J.; ZHANG, J. Recent global performance of the Climate Hazards group Infrared Precipitation (CHIRP) with Stations (CHIRPS). Journal of Hydrology, v. 591, p. 125284, 2020. Disponível em::<https://www.sciencedirect.com/science/article/pii/S0022169420307447? casa_to ken=xjABYhtVob0AAAAA:NFupbg7OFC4kkpJUTwMvkO_KA3V0iFHTEEww_8gzEpmB3fpphp7qTdwh1Xf9dewhcR8HtpalCTS.

doi.org/10.1016/j.jhydrol.2020.125284>. Acessado em: 19 set. 2023.

SILVA, E. P.; CAIONI, C.; DA SILVA, A. C. S.; OLIVEIRA, A. S. Adequação de modelo de erodibilidade de solos em nível de microbacia na borda sulamazônica. Revista de Ciências Agroambientais, v. 16, n. 2, p. 157-166, 2018. Disponível em: < https://periodicos.unemat.br/index.php/rcaa/article/view/2770. doi.org/10.5327/rcaa.v16i2.2770 >. Acessado em: 01 maç. 2021.

VALERIANO, M. M., ROSSETTI, D. F. Topodata: Brazilian full coverage refinement of SRTM data. Applied Geography (Sevenoaks), v. 32, p. 300-309, 2011. 
Disponível.em:.<https://www.sciencedirect.com/science/article/abs/pii/S01436228110 00786. doi.org/10.1016/j.apgeog.2011.05.004>. Acessado em: 04 maç. 2021.

WARD, A. Gravity Recovery and Climate Experiment (GRACE). NASA, v. 30, 2004. Disponível em:.<https://earthobservatory.nasa.gov/features/GRACE>. Acessado em: 16 maç. 2021.

WENG, W.; LUEDEKE, M. K.; ZEMP, D. C.; LAKES, T.; KROPP, J. P. Aerial and surface rivers: downwind impacts on water availability from land use changes in Amazonia. Hydrology and Earth System Sciences, v. 22, n. 1, p. 911-927, 2018. Disponível em: <https://hess.copernicus.org/articles/22/911/2018. doi.org/10.5194/hess-22-911-2018>. Acessado em: 07 set. 2020.

YI, L.; ZHANG, W.; WANG, K. Evaluation of heavy precipitation simulated by the WRF model using 4D-Var data assimilation with TRMM 3B42 and GPM IMERG over the Huaihe River Basin, China. Remote Sensing, v. 10, n. 4, p. 646, 2018. Disponível em: <https://www.mdpi.com/2072-4292/10/4/646. doi.org/10.3390/rs10040646>. Acessado em: 09 maç. 2021. 